\title{
A case report: Sjogren Larsson Syndrome
}

\begin{abstract}
Sjogren-Larsson Syndrome (SLS) is an autosomal recessive disorder with $100 \%$ penetrance. It has a characteristic set of clinical features, ichthyosis, spastic diplegia, and severe learning difficulties. To educate the patients about the treatment of dry skin and proper counseling regarding the genetic basis of disease are needed. The recent discoveries of the responsible biochemical abnormalities have explained the underlying links between the neurological and the dermatological manifestations. Its early diagnosis can help the physician to institute proper supportive treatment and possible curative gene therapy. Hence, ichthyosis cases must be investigated thoroughly.

We report a case of 19-year-old Indian male patient with clinical features favoring the rare disease, Sjogren-Larsson syndrome (SLS).
\end{abstract}

Keywords: Sjogren-larsson syndrome (SLS), fatty aldehyde dehydrogenase (FALDH), ichthyosiform

\section{Introduction}

Sjogren-Larsson Syndrome (SLS) is a rare autosomal recessive, neurocutaneous disorder. This rare syndrome harbors mutations in the ALDH3A2 gene located on chromosome $17 \mathrm{p} 11.2$ [1]. Abnormal lipid accumulation in the membranes of the skin and brain results in cutaneous and neurologic manifestations. SLS is characterized by generalized ichthyosis, mental retardation, and spastic paralysis. The syndrome presents at birth with ichthyosis ranging from fine scaling to generalized hyperkeratosis. Erythema may be the only finding noted at birth but it tends to clear gradually by 1 year of age. Colloidion like membrane is rarely seen. The ichthyosis manifests either as fine scale or large scale. Thickening of the stratum corneum without scale with pruritus can be the finding. Colour of thickened areas may be yellow to brown and have a lichenified appearance. Accentuated skin markings in flexural regions such as the sides and back of the neck, lower abdomen and flexures can be evident. The hair and nail are generally normal. Sweat function is well maintained in this disease [2]. Neurologic manifestations of spastic diplegia or tetraplegia and mental retardation develop at 2-3 years of age. These symptoms can be accompanied by speech defects and seizures. A characteristic ophthalmologic finding is the presence of glistening white dots in the macula of the retina. An eye finding manifests after 1 year of age and may not always be present in all patients. Non-specific histological findings of hyperkeratosis, papillomatosis, and acanthosis are seen on skin biopsy. Granular layer is mildly thickened in some situations. Electron microscopic examination of the skin shows lamellar membranous inclusions in the granular and cornified cells [3].

\section{Case Report}

We report a 19-year-old male patient, born of third degree consanguineous marriage, who presented with the dryness of skin all over the body since 15 days of age. History suggestive of pre-term delivery and delayed milestones was present. Although no appearance of blisters over the skin at any time since birth, the patient had difficulty in cognition and dimness of vision. The patient is attending school for the specially abled since 6 years of age. The patient had spastic diplegia since 2 years of age, for which he received treatment in the form of Botox injections without any signs of improvement. Individual or family history of atopy or epilepsy

\section{Desale Snehal 1,2,3, Gugle Anil ${ }^{3 *}$, Kote Rahul ${ }^{3}$, and Jadhav Vikrant ${ }^{3}$}

${ }^{1}$ Department of Dermatology, China Medical University Hospital, Taichung, 40402, Taiwan

${ }^{2}$ China Medical University, Taichung, 40402, Taiwan

${ }^{3}$ Department of Dermatology, MVP's Dr. Vasantrao Pawar Medical College, Nashik, Maharashtra, India

*Author for correspondence: dermatol.ndmvp25@gmail.com 
was absent. No history of similar complaints in any other sibling was reported by parents. General physical examination revealed spastic diplegic gait of jump knee pattern. Scrotum was full and normal in size. On Ocular examination, bilateral immature cataract was noticed (FIGURE 1). Intelligence quotient (IQ) was calculated to be 62 .

On dermatological examination, mountain ranges like hyperkeratotic scales were present all over the body except palms and soles. Ichthyosiform changes were noted on the upper trunk and extremities (FIGURES 1-3). Scales were found to be accentuated in flexures (FIGURES 4 and 5) which were dark brown in color. Scalp, hair, nails, oral mucosa and genital examination revealed to be normal. There was no evidence of ectropion, keratosis pilaris, or nerve deafness.

Magnetic Resonance Imaging (MRI) brain revealed metachromatic leukoencephalopathy and skin Biopsy (FIGURE 6A and 6B) demonstrated sparse superficial perivascular

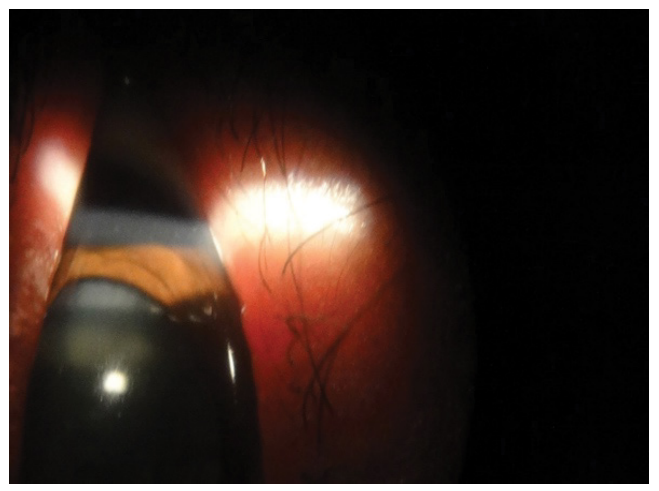

FIGURE 1. Immature cataract on slit lamp examination.

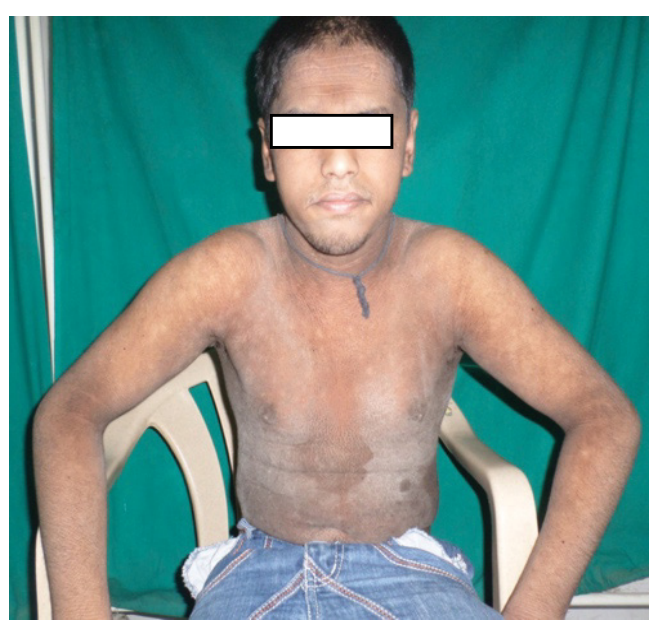

FIGURE 2. Icthyosiform changes with patchy xerosis over upper trunk and upper limbs .

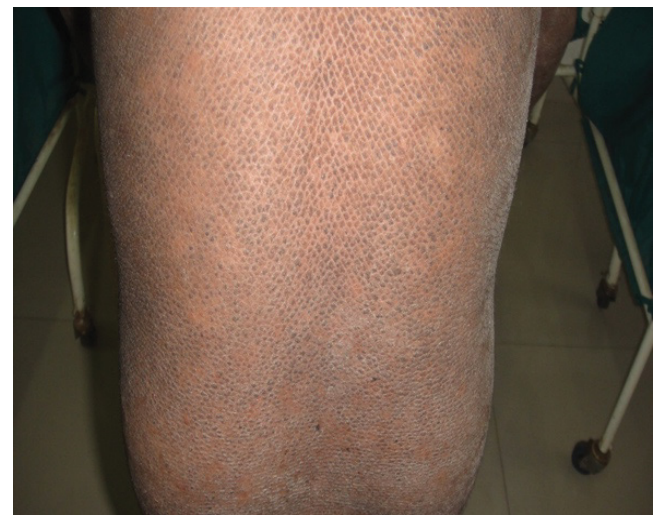

FIGURE 3. Icthyosiform changes over back (fish like scale appearance).

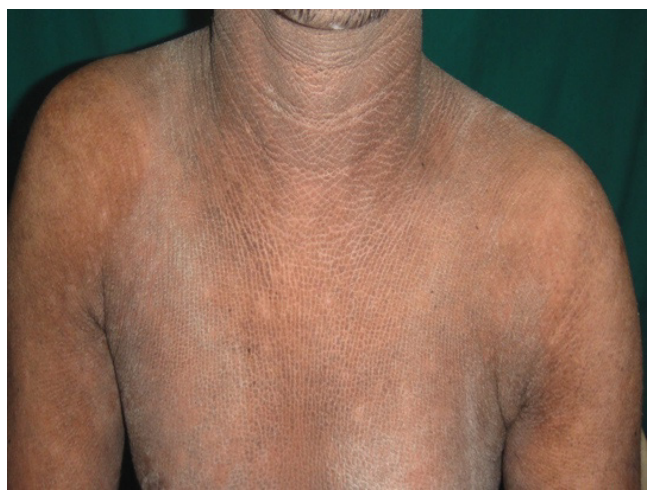

FIGURE 4. Accentuation of scales in flexural area (neck region).

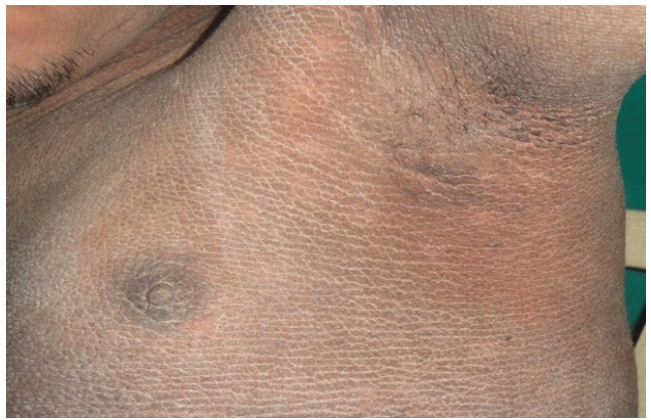

FIGURE 5. Accentuation of scales in flexures (axilla).
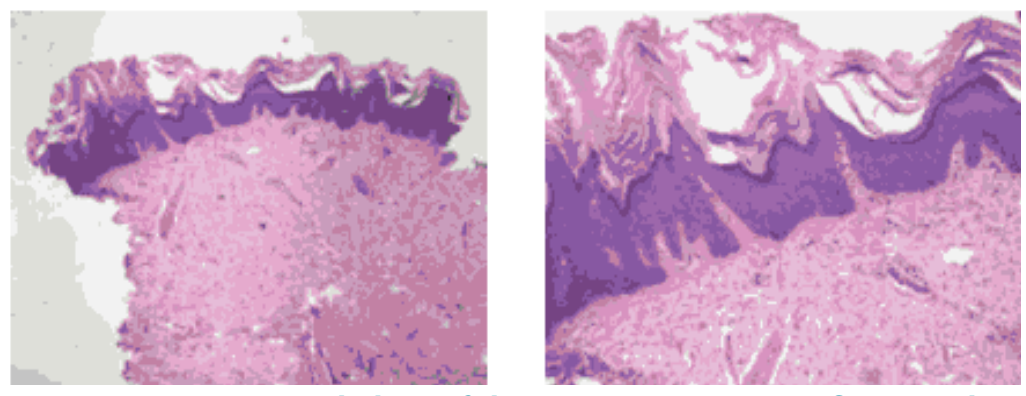

FIGURE 6. (a) Histopathology of skin (H/E stain 10X magnification); (b) Histopathology of skin (H/E stain 40X magnification).

lymphocytic infiltrate with slight epidermal hyperplasia. The granular layer was normal and 
the stratum corneum showed mild lamellated and compact orthohyperkeratosis. Histological findings, in this case, were suggestive of Ichthyosis.

The patient had a triad of clinical features of generalized ichthyosis, mental retardation, and spastic paralysis.

\section{Discussion}

Worldwide incidence of this rare condition is 0.4 per 100,000 [4]. Over 200 cases of this disease are reported worldwide. SLS was recognized as an inborn error of lipid metabolism. Deficiency of fatty aldehyde dehydrogenase could be the underlying cause for this syndrome. Rizzo et al. linked SLS to fatty alcohol: NAD oxidoreductase (FAO) deficiency. FAO is a complex enzyme with two separate proteins. These proteins catalyze the oxidation process of fatty alcohol to fatty acids via fatty aldehyde. Further research identified the Fatty Aldehyde Dehydrogenase (FALDH) activity in cultured fibroblasts from patients with SLS. Thus, FALDH was affected component of FAO in these group of SLS patients. Biochemically, FALDH is a microsomal enzyme that catalyzes the oxidation of medium and long-chain aliphatic aldehydes derived from various metabolisms in the body. These aldehydes may be by-products of metabolisms of phytanic acid, leukotriene B4, ether, fatty alcohol, and glycerolipids [5]. More than 70 mutations in ALDH3A2 have been identified in SLS patients. The several mutations are discovered such as amino acid substitutions, deletions, insertions, and splicing errors. An affected family carries a unique set of mutations but clinical variations do exist due to unknown environmental and or genetic factors [6]. ALDH10, recently named ALDH3A2 mutations (in the FALDH gene) confirmed the etiologic role of this enzyme. It also highlighted the importance of this pathway in normal desquamation [7-9]. Because of mutations, abnormal lipid accumulation in the membranes of brain and skin is postulated. Hence, a combination of cutaneous and neurologic symptoms is key to diagnosis. SLS constitutes the triad of generalized ichthyosis, mental retardation, and spastic paralysis.
Clinical findings are the main basis of diagnosis. Appropriate tests can be carried out for confirmation of the syndrome.

Rud's syndrome and Refsum's disease and other slowly progressive neurological disorders such as carbohydrate-deficient glycoprotein syndrome type 1, multiple sulphatase deficiency, neural lipid storage disorder, and mitochondrial disorders are to be differentiated from SLS [4]. We considered two provisional diagnoses, epidermolytic hyperkeratosis and ichthyosiform changes in SLS. The negative history of blisters in infancy and no histopathological changes in the granular and upper spinous layer and absence of suprabasal split ruled out epidermolytic hyperkeratosis. Congenital ichthyosis, epilepsy, sexual infantilism (only after puberty), polyneuritis, dwarfism and macrocytic anemia, clinical features of Rud's syndrome, were not present in this case. The patient had clinical features and histopathology favoring SLS. He had an additional ophthalmic finding of bilateral immature cataract, but the absence of a white glistening dot in the macula. Unfortunately, we couldn't define whether this cataract finding is a coincidental finding or part of this syndrome. As per literature glistening dot in the macula is observed as an eye involvement in $25 \%$ of SLS patients. The measurement of fatty aldehyde dehydrogenase in cultured fibroblasts from skin biopsies is necessary to confirm this rare disorder. Allele-specific polymerase chain reaction assay can detect known mutations [4]. Identification of abnormal urinary excretion of leukotriene B4 and its metabolites can also be useful [8-10]. Due to insufficient resources, we report the case on the basis of clinical, histopathological and MRI findings.

This case highlights a clinical triad of Sjogren Larsson syndrome. One should consider this rare but important differential diagnosis while dealing with a case of ichthyotic changes. Family history is important in such patients. Genetic counseling has a great role in these families. Gene therapy is a promising option for affected patients. As skin involves a larger surface area in this syndrome patient is likely to visit dermatology outpatient before neurology giving a first chance for dermatologists to diagnose it. 


\section{References}

Pavithran K, Karunakaran M, Palit A, et al. Disorders of keratinization. In: Valia RG, Valia AR, editors. IADVL Textbook of dermatology $3^{\text {rd }}$ ed. Mumbai: Bhalani Publishing House. 1002 (2008).

Jagell S, Liden S. Ichthyosis in the Sjogren-Larson syndrome. Clin. Genet. 21(4), 243-252 (1982).

Fleckman P, DiGiovanna JJ. The ichthyoses. In: Wolff K, Goldsmith LA, Katz SI, editors. $7^{\text {th }}$ ed. New York: McGraw-Hill, Dermatology in general medicine. 416-417 (2008).

Auada MP, Puzzi MB, Cintra MI, et al. Sjögren-larsson syndrome in Brazil is caused by a common c. $1108-1 \mathrm{G} \sim \mathrm{C}$ splice-site mutation in the ALDH3A2 gene. Br. J. Dermatol. 154(4), 770-773 (2006).

Rizzo WB. Sjogren-Larson syndrome: molecular genetics and biochemical pathogenesis of fatty aldehyde dehydrogenase deficiency. Mol. Genet. Metab. 90(1), 1-9 (2007).

Gordon N. Sjogren-larson syndrome. Dev. Med. Child. Neurol. 49(2), 152-154 (2007).

Borzyskowski M, Grant DB, Wells RS. Cushing's syndrome induced by topical steroids used for the treatment of non- bullous ichthyosiform erythroderma. Clin. Exp. Dermatol. 1(4), 337-342 (1976).

Willemsen MA, Ijlst L, Stijlen PM et al. Clinical, biochemical and molecular genetic characteristics of 19 patients with the Sjögren-Larsson syndrome. Brain. 124(7), 1426-1437 (2001).

Brandling-Bennett HA, Liang MG. What syndrome is this? Pediatr. Dermatol. 22(6), 569-571 (2005).

Willemsen MA, de Jong JG, van Domburg PH, et al. Defective inactivation of leukotriene B4 in patients with SjögrenLarsson syndrome. J. Pediatr. 136(2), 258260 (2000). 